\title{
SLC22 family of organic cation and anion transporters (version 2019.4) in the IUPHAR/BPS Guide to Pharmacology Database
}

\author{
Bruno Hagenbuch ${ }^{1}$
}

1. University of Kansas, USA

\begin{abstract}
The SLC22 family of transporters is mostly composed of non-selective transporters, which are expressed highly in liver, kidney and intestine, playing a major role in drug disposition. The family may be divided into three subfamilies based on the nature of the substrate transported: organic cations (OCTs), organic anions (OATs) and organic zwiterrion/cations (OCTN). Membrane topology is predicted to contain 12 TM domains with intracellular termini, and an extended extracellular loop at TM 1/2.
\end{abstract}

\section{Contents}

This is a citation summary for SLC22 family of organic cation and anion transporters in the Guide to Pharmacology database (GtoPdb). It exists purely as an adjunct to the database to facilitate the recognition of citations to and from the database by citation analyzers. Readers will almost certainly want to visit the relevant sections of the database which are given here under database links.

GtoPdb is an expert-driven guide to pharmacological targets and the substances that act on them. GtoPdb is a reference work which is most usefully represented as an on-line database. As in any publication this work should be appropriately cited, and the papers it cites should also be recognized. This document provides a citation for the relevant parts of the database, and also provides a reference list for the research cited by those parts.

Please note that the database version for the citations given in GtoPdb are to the most recent preceding version in which the family or its subfamilies and targets were substantially changed. The links below are to the current version. If you need to consult the cited version, rather than the most recent version, please contact the GtoPdb curators.

\section{Database links}

SLC22 family of organic cation and anion transporters

http://www.guidetopharmacology.org/GRAC/FamilyDisplayForward?familyld=146

Organic cation transporters (OCT)

http://www.guidetopharmacology.org/GRAC/FamilyDisplayForward?familyld=196

Transporters

OCT1 (Organic cation transporter 1)

http://www.guidetopharmacology.org/GRAC/ObjectDisplayForward?objectld=1019

OCT2(Organic cation transporter 2) 
http://www.guidetopharmacology.org/GRAC/ObjectDisplayForward?objectld=1020 OCT3(Organic cation transporter 3)

http://www.guidetopharmacology.org/GRAC/ObjectDisplayForward?objectld=1021 Organic zwitterions/cation transporters (OCTN)

http://www.guidetopharmacology.org/GRAC/FamilyDisplayForward?familyld=197

Transporters

OCTN1(Organic cation/carnitine transporter 1)

http://www.guidetopharmacology.org/GRAC/ObjectDisplayForward?objectld=1022

OCTN2(Organic cation/carnitine transporter 2)

http://www.guidetopharmacology.org/GRAC/ObjectDisplayForward?objectld=1023

CT2(Carnitine transporter 2)

http://www.guidetopharmacology.org/GRAC/ObjectDisplayForward?objectld=1024

Organic anion transporters (OATs)

http://www.guidetopharmacology.org/GRAC/FamilyDisplayForward?familyld=198

Transporters

OAT1(Organic anion transporter 1)

http://www.guidetopharmacology.org/GRAC/ObjectDisplayForward?objectld=1025 OAT2(Organic anion transporter 2)

http://www.guidetopharmacology.org/GRAC/ObjectDisplayForward?objectld=1026

OAT3(Organic anion transporter 3)

http://www.guidetopharmacology.org/GRAC/ObjectDisplayForward?objectld=1027

Organic anion transporter 4

http://www.guidetopharmacology.org/GRAC/ObjectDisplayForward?objectld=1030

OAT5(Organic anion transporter 5)

http://www.guidetopharmacology.org/GRAC/ObjectDisplayForward?objectld=1029

OAT4(Organic anion transporter 7)

http://www.guidetopharmacology.org/GRAC/ObjectDisplayForward?objectld=1028

Urate transporter

http://www.guidetopharmacology.org/GRAC/FamilyDisplayForward?familyld=199

Transporters

URAT1(Urate anion exchanger 1)

http://www.guidetopharmacology.org/GRAC/ObjectDisplayForward?objectld=1031

Orphan or poorly characterized SLC22 family members

http://www.guidetopharmacology.org/GRAC/FamilyDisplayForward?familyld=200

Transporters

ORCTL3(Organic cation transporter-like 3)

http://www.guidetopharmacology.org/GRAC/ObjectDisplayForward?objectld=1032

ORCTL4(Organic cation transporter-like 4)

http://www.guidetopharmacology.org/GRAC/ObjectDisplayForward?objectld=1033

FLIPT1(Fly-like putative transporter 1)

http://www.guidetopharmacology.org/GRAC/ObjectDisplayForward?objectld=1034

BOIT(Brain-type organic cation transporter)

http://www.guidetopharmacology.org/GRAC/ObjectDisplayForward?objectld=1035

ORCTL2(Organic cation transporter-like 2)

http://www.guidetopharmacology.org/GRAC/ObjectDisplayForward?objectld=1036

OAT6

http://www.guidetopharmacology.org/GRAC/ObjectDisplayForward?objectld=1037

SLC22A23

http://www.guidetopharmacology.org/GRAC/ObjectDisplayForward?objectld=1038

SLC22A24 
http://www.guidetopharmacology.org/GRAC/ObjectDisplayForward?objectld=1039

UST6

http://www.guidetopharmacology.org/GRAC/ObjectDisplayForward?objectld=1040

solute carrier family 22 member 31

http://www.guidetopharmacology.org/GRAC/ObjectDisplayForward?objectld=2557

Atypical SLC22B subfamily

http://www. guidetopharmacology.org/GRAC/FamilyDisplayForward?familyld=859

Transporters

synaptic vesicle glycoprotein $2 \mathrm{~A}$

http://www.guidetopharmacology.org/GRAC/ObjectDisplayForward?objectld=2634

\section{References}

1. Burns CM and Wortmann RL. (2011) Gout therapeutics: new drugs for an old disease.Lancet 377: 165-77 [PMID:20719377]

2. Cha SH, Sekine T, Kusuhara H, Yu E, Kim JY, Kim DK, Sugiyama Y, Kanai Y and Endou H. (2000) Molecular cloning and characterization of multispecific organic anion transporter 4 expressed in the placenta. J. Biol. Chem. 275: 4507-12 [PMID:10660625]

3. Diaz-Torné C, Perez-Herrero N and Perez-Ruiz F. (2015) New medications in development for the treatment of hyperuricemia of gout. Curr Opin Rheumatol 27: 164-9 [PMID:25603039]

4. Enomoto A, Kimura H, Chairoungdua A, Shigeta Y, Jutabha P, Cha SH, Hosoyamada M, Takeda M, Sekine T and Igarashi T et al.. (2002) Molecular identification of a renal urate anion exchanger that regulates blood urate levels. Nature 417: 447-52 [PMID:12024214]

5. Gorboulev V, Ulzheimer JC, Akhoundova A, Ulzheimer-Teuber I, Karbach U, Quester S, Baumann C, Lang F, Busch AE and Koepsell $\mathrm{H}$. (1997) Cloning and characterization of two human polyspecific organic cation transporters. DNA Cell Biol. 16: 871-81 [PMID:9260930]

6. Gründemann D, Liebich G, Kiefer N, Köster S and Schömig E. (1999) Selective substrates for nonneuronal monoamine transporters. Mol. Pharmacol. 56: 1-10 [PMID:10385678]

7. Gründemann D, Schechinger B, Rappold GA and Schömig E. (1998) Molecular identification of the corticosterone-sensitive extraneuronal catecholamine transporter. Nat. Neurosci. 1: 349-51 [PMID:10196521]

8. Hamada T, Ichida K, Hosoyamada M, Mizuta E, Yanagihara K, Sonoyama K, Sugihara S, Igawa O, Hosoya T and Ohtahara A et al.. (2008) Uricosuric action of losartan via the inhibition of urate transporter 1 (URAT 1) in hypertensive patients. Am. J. Hypertens. 21: 1157-62 [PMID:18670416]

9. Ichida K, Hosoyamada M, Kimura H, Takeda M, Utsunomiya $Y$, Hosoya T and Endou H. (2003) Urate transport via human PAH transporter hOAT1 and its gene structure. Kidney Int. 63: 143-55 [PMID:12472777]

10. Kenda BM, Matagne AC, Talaga PE, Pasau PM, Differding E, Lallemand BI, Frycia AM, Moureau FG, Klitgaard HV and Gillard MR et al.. (2004) Discovery of 4-substituted pyrrolidone butanamides as new agents with significant antiepileptic activity. J. Med. Chem. 47: 530-49 [PMID:14736235]

11. Kimura H, Takeda M, Narikawa S, Enomoto A, Ichida K and Endou H. (2002) Human organic anion transporters and human organic cation transporters mediate renal transport of prostaglandins. J. Pharmacol. Exp. Ther. 301: 293-8 [PMID:11907186]

12. Klitgaard $\mathrm{H}$ and Verdru P. (2007) Levetiracetam: the first SV2A ligand for the treatment of epilepsy.Expert Opin Drug Discov 2: 1537-45 [PMID:23484603]

13. Kusuhara H, Sekine T, Utsunomiya-Tate N, Tsuda M, Kojima R, Cha SH, Sugiyama Y, Kanai $Y$ and Endou $\mathrm{H}$. (1999) Molecular cloning and characterization of a new multispecific organic anion transporter from rat brain. J. Biol. Chem. 274: 13675-80 [PMID:10224140]

14. Longo N, Frigeni M and Pasquali M. (2016) Carnitine transport and fatty acid oxidationBiochim. Biophys. Acta 1863: 2422-35 [PMID:26828774]

15. Löscher W, Gillard M, Sands ZA, Kaminski RM and Klitgaard H. (2016) Synaptic Vesicle Glycoprotein 2A 
Ligands in the Treatment of Epilepsy and Beyond. CNS Drugs 30: 1055-1077 [PMID:27752944]

16. Mendoza-Torreblanca JG, Vanoye-Carlo A, Phillips-Farfán BV, Carmona-Aparicio L and Gómez-Lira G. (2013) Synaptic vesicle protein 2A: basic facts and role in synaptic function. Eur. J. Neurosci. 38: 3529-39 [PMID:24102679]

17. Nicolas JM, Hannestad J, Holden D, Kervyn S, Nabulsi N, Tytgat D, Huang Y, Chanteux H, Staelens L and Matagne A et al.. (2016) Brivaracetam, a selective high-affinity synaptic vesicle protein 2A (SV2A) ligand with preclinical evidence of high brain permeability and fast onset of action. Epilepsia 57: 201-9 [PMID:26663401]

18. Noyer M, Gillard M, Matagne A, Hénichart JP and Wülfert E. (1995) The novel antiepileptic drug levetiracetam (ucb L059) appears to act via a specific binding site in CNS membranes. Eur. J. Pharmacol. 286: 137-46 [PMID:8605950]

19. Pao SS, Paulsen IT and Saier Jr MH. (1998) Major facilitator superfamily Microbiol. Mol. Biol. Rev. 62: 134 [PMID:9529885]

20. Perland E, Bagchi S, Klaesson A and Fredriksson R. (2017) Characteristics of 29 novel atypical solute carriers of major facilitator superfamily type: evolutionary conservation, predicted structure and neuronal co-expression. Open Biol 7: [PMID:28878041]

21. Perland E and Fredriksson R. (2017) Classification Systems of Secondary Active Transporters.Trends Pharmacol. Sci. 38: 305-315 [PMID:27939446]

22. Reddy VS, Shlykov MA, Castillo R, Sun El and Saier Jr MH. (2012) The major facilitator superfamily (MFS) revisited. FEBS J. 279: 2022-35 [PMID:22458847]

23. Shu Y, Sheardown SA, Brown C, Owen RP, Zhang S, Castro RA, lanculescu AG, Yue L, Lo JC and Burchard EG et al.. (2007) Effect of genetic variation in the organic cation transporter 1 (OCT1) on metformin action. J. Clin. Invest. 117: 1422-31 [PMID:17476361]

24. Takeda M, Babu E, Narikawa S and Endou H. (2002) Interaction of human organic anion transporters with various cephalosporin antibiotics. Eur. J. Pharmacol. 438: 137-42 [PMID:11909604]

25. Vogl C, Tanifuji S, Danis B, Daniels V, Foerch P, Wolff C, Whalley BJ, Mochida S and Stephens GJ. (2015) Synaptic vesicle glycoprotein $2 A$ modulates vesicular release and calcium channel function at peripheral sympathetic synapses. Eur. J. Neurosci. 41: 398-409 [PMID:25484265]

26. von Rosenstiel P. (2007) Brivaracetam (UCB 34714). Neurotherapeutics 4: 84-7 [PMID:17199019]

27. Youngblood GL and Sweet DH. (2004) Identification and functional assessment of the novel murine organic anion transporter Oat5 (Slc22a19) expressed in kidney. Am. J. Physiol. Renal Physiol. 287: F23644 [PMID:15068970]

28. Yu Z, Fong WP and Cheng CH. (2007) Morin (3,5,7,2',4'-pentahydroxyflavone) exhibits potent inhibitory actions on urate transport by the human urate anion transporter (hURAT1) expressed in human embryonic kidney cells. Drug Metab. Dispos. 35: 981-6 [PMID:17325024]

29. Zhang L, Schaner ME and Giacomini KM. (1998) Functional characterization of an organic cation transporter (hOCT1) in a transiently transfected human cell line (HeLa). J. Pharmacol. Exp. Ther. 286: 35461 [PMID:9655880]

30. Zhu HJ, Appel DI, Gründemann D and Markowitz JS. (2010) Interaction of organic cation transporter 3 (SLC22A3) and amphetamine. J. Neurochem. 114: 142-9 [PMID:20402963] 